\section{Cost of illness of spinal muscular atrophy (SMA) in Italy}

\author{
Andrea Marcellusi ',2 (D), Chiara Bini', Jacopo Casiraghi³, \\ Francesco D'Ambrosio', Maria A. Rotundo' (D), Anita Pallara ${ }^{4}$ \\ and Francesco S. Mennini ',2 on behalf of Famiglie SMA Onlus \\ (Parents for Research on Spinal Muscular Atrophy, Nonprofit \\ Organization), Italy.
}

Global \& Regional Health Technology Assessment

Volume 2019: 1-12

(C) The Author(s) 2019

Article reuse guidelines: sagepub.com/journals-permissions DOI: 10.1 I77/22842403।9867662 journals.sagepub.com/home/grh (3)SAGE

\begin{abstract}
The objective of this study was to estimate the indirect and direct non-health costs associated with spinal muscular atrophy (SMA), a disease that burdens the daily life of adults, children and their families in Italy. In order to develop the economic model, a multidisciplinary group of researchers was created to prepare and computerize a questionnaire, which was promoted by SMA families in collaboration with the Economic Evaluation and Heath Technology Assessment center at the University of Rome Tor Vergata. The analysis envisaged a first phase for implementing and validating the questionnaire by the multidisciplinary group. Subsequently, the questionnaire was computerized and sent out to be completed through all the association's distribution channels. The social channels and specific mailing lists were limited exclusively to SMA families. To achieve the sample number required by the research protocol, data collection began on January 8, 2018, and closed on April 15, 2018. Finally, all the data were analyzed using the economic model in order to estimate the average costs per patient. The questionnaire was able to identify a sample of I I 8 families (22.88\% SMA I, $48.31 \%$ SMA II, $28.81 \%$ SMA III). The average age of the patients was 18.49 years (average age at diagnosis 2.88 years) with more females $(55,08 \%)$ in the total respondents, taking into account a $4.24 \%$ rate of non-respondents. The economic model estimated an average annual cost per patient with SMA of €I 5.37I,4I (€ I 7.683,85 for SMA I, €I5.974,78 for SMA II and $€ I 2.523,52$ for SMA III). Of these costs, about $52 \%$ were attributable to indirect costs associated with caregivers, $15 \%$ for indirect costs associated with the patient and $4 \%$ for social security costs. A total of $17 \%$ was attributable to the direct costs incurred by the patient and I $2 \%$ was attributable to the direct costs incurred by the Italian National Health Service (SSN). To our knowledge, this survey represents the first nationwide analysis estimating the costs incurred by families for the management of SMA. This study highlights the need for specific policies to support families who must live with the disease, not only from the standpoint of their compromised quality of life but also due to the significant economic burden imposed by the disease.
\end{abstract}

\title{
Keywords
}

Cost of illness, indirect costs, spinal muscular atrophy, economic burden of disease

\footnotetext{
'Economic Evaluation and HTA (CEIS-EEHTA) - IGF Department, Faculty of Economics, University of Rome Tor Vergata, Italy Institute for Leadership and Management in Health, Kingston University London, London, UK

${ }^{3}$ Neurorehabilitation Unit, University of Milan, Nemo Clinical Center, Milan; and Famiglie SMA Onlus (Parents for Research on Spinal Muscular Atrophy, Nonprofit Organization), Italy
}

\footnotetext{
${ }^{4}$ Famiglie SMA Onlus (Parents for Research on Spinal Muscular Atrophy, Nonprofit Organization), Italy

Corresponding author:

Dr. Andrea Marcellusi, Economic Evaluation and HTA (EEHTA) Faculty of Economics, University of Rome Tor Vergata, Via Columbia 2, Rome, 00133, Italy.

Email: andrea.marcellusi@uniroma2.it
} 


\section{Introduzione}

L'atrofia muscolare spinale (spinal muscular atrophy, SMA) è una malattia neuromuscolare genetica rara del sistema nervoso che si manifesta quando il soggetto eredita da entrambi i genitori mutazioni nel gene responsabile della patologia. È la principale causa genetica di mortalità infantile, con un'incidenza pari a $1 / 10.000$ nati vivi. ${ }^{1}$

È caratterizzata dalla progressiva degenerazione dei motoneuroni, le cellule nervose del midollo spinale, il cui compito è di controllare il movimento. Ne consegue, per i soggetti che ne sono affetti, un progressivo indebolimento e atrofia muscolare che interessa, in particolar modo, gli arti inferiori e i muscoli respiratori. I pazienti affetti da SMA vanno dunque incontro a un progressivo peggioramento della propria qualità di vita, che oltre a comportare l'impossibilità di svolgere un'attività lavorativa o di svolgere un'attività lavorativa al 100\% della propria capacità produttiva, in alcuni casi comporta anche l'impossibilità di svolgere le semplici attività quotidiane. Per questo motivo, la maggior parte dei pazienti ha bisogno di assistenza da parte dei propri caregivers, i quali potrebbero incorrere nell'impossibilità di svolgere, totalmente o parzialmente, la propria attività lavorativa.

La SMA si presenta con uno spettro di cinque sottotipi, con diversi gradi di severità, in base all'età di insorgenza dei sintomi e alla massima funzione motoria raggiunta, in accordo alla classificazione dell'International SMA Consortium (ISMAC):2,3

- SMA di tipo 0: insorgenza durante la vita fetale, con movimenti fetali attivi ridotti o del tutto assenti. Debolezza ed ipotonia severe, grave insufficienza respiratoria. Aspettativa di vita di poche settimane.

- SMA di tipo I: caratterizzata da insorgenza dei sintomi prima dei sei mesi di età e aspettativa di vita inferiore ai due anni senza supporto respiratorio; i bambini non raggiungono la capacità di sedersi o girarsi in modo autonomo.

- SMA di tipo II: esordio dei sintomi tra i 7 ed i 18 mesi di vita. I soggetti affetti possono sedersi in modo indipendente, ma non sono mai in grado di camminare.

- SMA di tipo III: insorgenza dei sintomi dopo i 18 mesi. I soggetti affetti sono in grado di stare in piedi e di camminare in modo indipendente.

- SMA di tipo IV: insorgenza nell'età adulta; si tratta della forma più lieve con generali difficoltà motorie.

La patologia in oggetto è ancora oggi incurabile, ma recenti ricerche cliniche sperimentali hanno individuato possibili terapie innovative in grado di ridurre la progressione della malattia e migliorare la qualità di vita dei pazienti (e conseguentemente dei caregivers). Ovviamente, alle scoperte di cure innovative, soprattutto nel caso di patologie rare, si affiancano necessità di carattere economico e di investimento per l'acquisto di tali terapie da parte del Servizio Sanitario Nazionale (SSN). In questo contesto, emerge il bisogno di analisi economiche dettagliate che permettano di comprendere la reale portata della malattia in oggetto poiché a un basso livello di qualità di vita e a una bassa aspettativa di vita risultano associati ingenti costi per il SSN, per le famiglie e per la società.

Ad oggi, sia a livello nazionale che internazionale, le informazioni economiche sui costi generati dalla malattia sono molto scarsi. In Germania, uno studio costo della malattia (cost of illness, COI) condotto attraverso la somministrazione di specifici questionari a pazienti e famiglie SMA, ${ }^{4}$ ha stimato un carico economico complessivo nella prospettica sociale tedesca superiore a $€ 106$ milioni annui. Di questi, il 20\% sono rappresentati dai costi diretti sanitari, il 58\% da costi diretti non sanitari e oltre il $22 \%$ sono rappresentati da costi indiretti legati alla perdita di produttività dei pazienti e delle loro famiglie. ${ }^{4}$

L'obiettivo del presente lavoro è stato quello di stimare il peso economico e sociale dell'SMA in Italia attraverso lo sviluppo di un modello statistico-economico in grado di calcolare i costi riconducibili alla patologia in oggetto. Le categorie di costo considerate nell'analisi sono state le seguenti:

- costi diretti, associati alla patologia in termini di diagnosi, trattamento e gestione della malattia e possono risultare a carico del SSN o a carico del paziente (al netto del rimborso statale);

- costi indiretti, associati alla riduzione o alla totale perdita di produttività sul lavoro del paziente e dei suoi caregivers e calcolati in termini di assenteismo, presenteismo e disoccupazione.

Ulteriore obiettivo dell' analisi è stato quello di dimostrare l'importanza dell'inclusione dei costi indiretti negli studi diCOI, in quanto essi spesso rappresentano una percentuale significativa del peso complessivo di una patologia e la loro esclusione potrebbe portare a sottostimare il peso economico complessivo della malattia.

Lo studio di COI rappresenta uno strumento molto utile al fine di ottenere un miglioramento qualitativo delle decisioni in sanità, soprattutto se affrontati da una prospettiva sociale. Gli studi di COI mettono in evidenza tutti quei costi che spesso vengono trascurati e che sono rappresentati dai costi a carico dei pazienti e dai costi a carico della società.

\section{Metodi}

Al fine di stimare i costi diretti e indiretti associati alla SMA e di colmare il gap informativo esistente, è stata effettuata una indagine cross-section con campionamento trasversale attraverso la somministrazione di questionari 


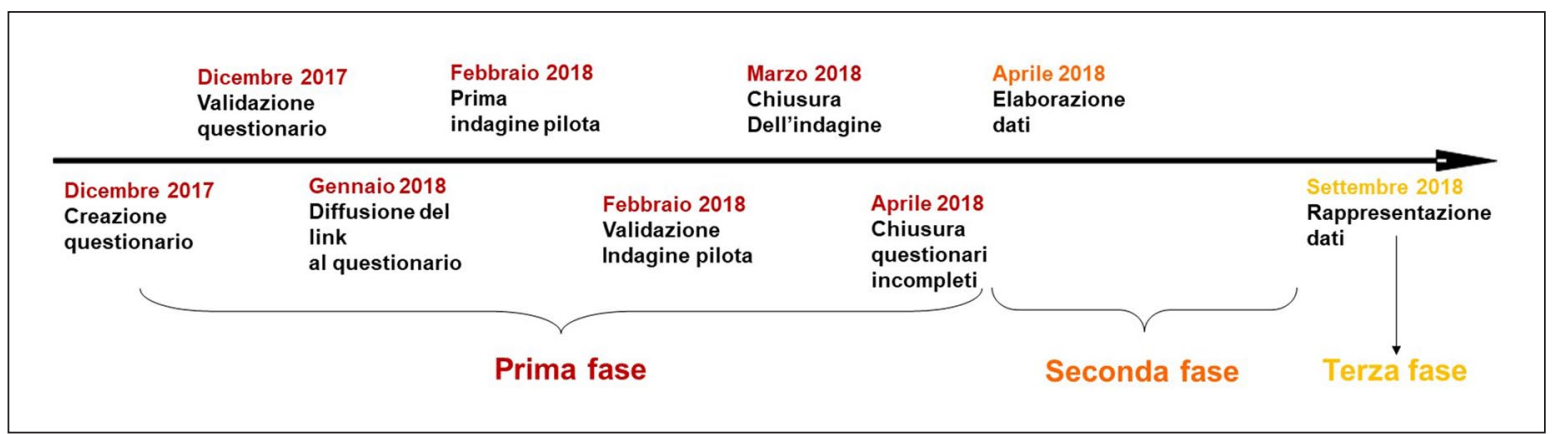

Figura I. Fasi dello studio.

on-line a un gruppo di famiglie afferenti all'associazione pazienti Famiglie SMA. L'indagine è stata sviluppata seguendo differenti passi metodologici riassunti in Figura 1. Come primo passo è stato sviluppato un questionario ad hoc sulla patologia in analisi considerando quanto già presente in letteratura. ${ }^{5,6} \mathrm{La}$ validazione del questionario, avvenuta in collaborazione con il comitato scientifico dell'zssociazione Famiglie SMA, è stata completata a Dicembre 2017 e a inizio Gennaio 2018 e si è provveduto all'invio dei questionari attraverso i canali comunicativi dell'associazione Famiglie SMA. Alle famiglie intervistate è stato inviato un link che riportava un questionario anonimo compilabile on-line.

L'indagine si è conclusa al raggiungimento della numerosità campionaria individuata tramite una stima intervallare definita secondo la rappresentatività rispetto al numero di pazienti prevalenti in Italia (circa 850 pazienti $^{7}$ ). In particolare, non avendo a disposizione dati riferiti all'end-point principale dell'indagine (costi diretti e indiretti sostenuti dai pazienti SMA), si è ritenuta accettabile una stima minima di 85 pazienti intervistati $(10 \%$ della popolazione prevalente) e un massimo di 127 pazienti (15\% della popolazione prevalente).

Al completamento dei primi 27 questionari (avvenuto a Febbraio 2018) è stata effettuata una prima analisi pilota con l'obiettivo di verificare eventuali incongruenze con le risposte alle diverse domande del questionario. La raccolta dei questionari si è chiusa ad Aprile 2018 e i primi risultati presentati a Settembre dello stesso anno (Figura 1).

Il questionario era composto da 43 domande (36 se il rispondente era il caregiver che si occupa del paziente) e suddiviso in sei sezioni:

- Caratteristiche rispondenti: contenente domande finalizzate a conoscere la persona che compila il questionario e la storia di malattia della famiglia;

- Area conoscitiva: finalizzata all'ottenimento di informazioni più dettagliate sulla vita quotidiana delle persone affette da SMA;

- Area anagrafica caregivers: contenente domande circa aspetti e caratteristiche dei caregivers;
- Ambito sanitario: contenente domande utili per la comprensione del percorso diagnostico e terapeutico seguito dal paziente e i costi a esso associati;

- Sgravi e benefici economici: contenente domande finalizzate all'analisi dell'applicazione di leggi a tutela del paziente ed eventuali contributi economici ricevuti dallo Stato o da altri Enti pubblici;

- Utilizzo di ausili e attrezzature mediche; contenente domande circa l'utilizzo di ausili e attrezzature mediche ed eventuali costi sostenuti dal paziente e dalla famiglia per tale utilizzo.

Ai rispondenti è stato chiesto di fornire informazioni di carattere sociodemografico e relative al consumo di risorse (a carico proprio o del SSN) relativo in particolare all'ultimo anno dalla data di compilazione. In alcuni casi, al fine di evitare l'effetto memoria, le domande facevano riferimento agli ultimi tre mesi vissuti con la malattia ipotizzando poi un andamento lineare del fenomeno nel corso del tempo (dettagli in appendice).

I questionari sono stati auto-compilati in forma anonima dai pazienti maggiorenni e dalle loro famiglie, mentre per i pazienti minorenni la compilazione del questionario è stata affidata al caregiver. Le domande riguardanti le caratteristiche professionali ed economiche del paziente sono state rivolte solamente ai pazienti maggiorenni.

Ciascuna voce di costo (diretto o indiretto) è stata calcolata a livello di singolo paziente in relazione alle caratteristiche di quest'ultimo e alle risposte fornite. Tali voci di costo sono state poi sommate per tipologia; dividendo ciascuna somma per il numero di rispondenti al questionario è stato possibile ottenere il costo medio per paziente affetto da SMA per ciascuna voce di costo.

Il peso economico complessivo della SMA a livello nazionale è stato ottenuto attribuendo il costo medio per paziente, ottenuto dal campione, al numero dei pazienti affetti da SMA.

Al fine di quantificare i costi diretti, ai rispondenti è stato chiesto di riportare il numero di volte in cui avessero usufruito, nell'ultimo anno, di determinati servizi e prestazioni sanitari. Tale numero è stato poi moltiplicato 
per la tariffa associata a ciascuna prestazione, reperita all'interno del Nomenclatore Tariffario Nazionale. ${ }^{8}$

Per la stima dei costi indiretti è stato impiegato il metodo del capitale umano (human capital approach): ciò equivale a misurare la mancata produttività, di un paziente o di un caregiver, in termini di deficit di guadagni dovuti alla perdita di produzione causati dalla malattia. In particolare, le stime dei costi indiretti sopra definiti sono state effettuate tenendo in considerazione le seguenti condizioni occupazionali del paziente o del caregiver:

- assenteismo: la mancata produttività dovuta all'assenza dal lavoro da parte del paziente a causa della malattia o del caregiver a causa della prestazione di assistenza;

- presenteismo: relativo a una riduzione della capacità produttiva del paziente che si reca a lavoro ma, a causa della malattia, produce meno rispetto agli standard;

- disoccupazione: la mancata occupazione (e quindi mancata produzione) del paziente causata dalla malattia o del caregiver a causa della prestazione di assistenza.

I costi indiretti per assenteismo e presenteismo sono stati stimati moltiplicando il numero medio di giornate lavorative perse, o con una ridotta capacità produttiva (presenteismo), in un anno, per il reddito medio giornaliero del paziente (o del caregiver) occupato.

Il numero medio di giornate lavorative perse o con ridotta capacità produttiva è stato ottenuto tramite apposite domande presenti nel questionario attraverso le quali agli intervistati veniva richiesto di riportare il numero di giornate lavorative perse o con una ridotta produttività sul lavoro negli ultimi tre mesi; il numero di giornate lavorative perse o con una capacità produttiva ridotta in un anno è stato ottenuto, per i soli pazienti o caregiver occupati, assumendo un andamento lineare nel tempo.

Il reddito medio giornaliero è stato ottenuto a partire dal reddito medio annuo dichiarato dai pazienti o dai caregiver occupati appartenenti al campione e assumendo 250 giorni lavorativi in un anno. ${ }^{9}$

Per la stima dei costi previdenziali, l'analisi ha preso in considerazione la Legge 289/90 e la Legge 104/92; in particolare con riferimento alla prima, relativa all'indennità di frequenza e che prevede che venga corrisposto un contributo economico mensile pari a $€ 279,47$ (per l'anno 2017) agli invalidi civili minori d'età, si è assunta una fruizione minima del contributo (pari a nove mesi) per i pazienti minorenni il cui caregiver ne avesse dichiarato la fruizione. Relativamente alle Legge 104/92 la quale prevede che il caregiver del paziente la cui invalidità sia stata accertata usufruisca di quattro giorni al mese di permesso retribuito per l'assistenza fornita al proprio familiare, la quantificazione della perdita di produttività è stata effettuata considerando il reddito giornaliero del rispondente.

La Tabella 1 riporta i parametri di costo unitari utilizzati per le stime del modello e applicate alle frequenze registrate nel campione utilizzato.

Le variabili qualitative sono state riportate in termini di percentuali sul totale arruolati mentre le variabili quantitative come media e deviazione standard. Al fine di individuare eventuali differenze statisticamente significative dei costi tra due gruppi è stato impiegato il rispettivamente il test di Mann-Whitney, mentre per le differenze tra più gruppi sono stati impiegati il modello lineare generalizzato con distribuzione Gamma e la funzione $\log$ come funzione link ed il test di Test di Kruskal-Wallis. ${ }^{11}$

\section{Risultati}

Delle 300 famiglie afferenti all'associazione Famiglie SMA al momento dell'invio del questionario, 122 soggetti (40\% degli arruolabili) hanno risposto al questionario on-line. Di queste, 118 famiglie (39\% degli arruolabili e $97 \%$ dei rispondenti) hanno risposto a oltre il $50 \%$ delle domande e sono state incluse nell'analisi. In Tabella 2 sono riportate le caratteristiche generali dei pazienti appartenenti al campione analizzato.

L'età media dei pazienti appartenenti al campione è risultata pari a circa 18 anni (range 0-74). Il campione è risultato costituito per il $67 \%$ da pazienti minorenni e per il $55 \%$ da pazienti di sesso femminile.

La tipologia di SMA più frequente all'interno del campione è risultata quella di tipo II, con una percentuale pari a circa il $48 \%$ dei rispondenti, mentre con riferimento alle tipologie I e III la percentuale di pazienti è risultata rispettivamente pari a circa il $23 \%$ ed il $29 \%$ del campione. In Tabella 3 sono riportati ulteriori parametri, relativi ai pazienti e ai caregivers, utili ai fini della stima dei costi indiretti.

Le informazioni relative allo stato lavorativo dei pazienti maggiorenni appartenenti al campione sono riportate in Tabella 3. Tra i pazienti occupati (pari al circa il $33,3 \%$ del campione), il $53,6 \%$ ha dichiarato di aver perso giornate di lavoro a causa della malattia mentre il $23,1 \%$ ha dichiarato di aver sperimentato una riduzione della propria capacità produttiva sul lavoro a causa della malattia. All'interno del questionario ai pazienti è stato richiesto di riportare una percentuale che potesse quantificare approssimativamente la propria riduzione della capacità produttiva sul lavoro a causa della malattia. Tenendo conto dei soli pazienti occupati, tale riduzione è risultata mediamente pari al 28,5\%. La percentuale dei pazienti affetti da SMA non occupati a causa della malattia è risultata pari al 15,4 . Tale metodo è stato precedentemente utilizzato in studi simili volti alla valutazione dei costi indiretti mediante la somministrazione di un questionario. ${ }^{12}$ 
Tabella I. Parametri di costo utilizzati ai fini della stima dei costi diretti e indiretti.

\begin{tabular}{|c|c|c|}
\hline Prestazione & Tariffa & Fonte \\
\hline $\begin{array}{l}\text { Risonanza magnetica nucleare }(\mathrm{RM}) \text { del cervello e } \\
\text { del tronco encefalico (cod. 88.98.I) }\end{array}$ & $€ 166,5$ & {$[10]$} \\
\hline Elettrocardiogramma (cod. 89.52) & $€ \mid I, 62$ & {$[10]$} \\
\hline $\begin{array}{l}\text { Emocromo: Hb, GR, GB, HCT, PLT, IND. } \\
\text { DERIV., F.L. (cod.90.62.2) }\end{array}$ & $€ 3,17$ & {$[10]$} \\
\hline $\begin{array}{l}\text { Neurologo/Neuropsichiatra infantile (cod. } \\
\text { 89.7) }\end{array}$ & $€ 20,66$ & {$[10]$} \\
\hline Pediatra (cod. 89.7) & $€ 20,66$ & {$[10]$} \\
\hline Ortopedico specialista (cod. 89.7) & $€ 20,66$ & {$[10]$} \\
\hline Cardiologo (cod. 89.7) & $€ 20,66$ & {$[10]$} \\
\hline Pneumologo (cod. 89.7) & $€ 20,66$ & {$[10]$} \\
\hline Genetista (cod. 89.7) & $€ 20,66$ & {$[10]$} \\
\hline Fisioterapista (cod. 89.7) & $€ 20,66$ & {$[10]$} \\
\hline Logopedista (cod. 89.7) & $€ 20,66$ & {$[10]$} \\
\hline Terapista occupazionale (cod. 89.7) & $€ 20,66$ & {$[10]$} \\
\hline Terapista respiratorio (cod. 89.7) & $€ 20,66$ & {$[10]$} \\
\hline Fisiatra (cod. 89.7) & $€ 20,66$ & {$[10]$} \\
\hline Psichiatra (cod. 89.7) & $€ 20,66$ & {$[10]$} \\
\hline Otorinolaringoiatra (cod. 89.7) & $€ 20,66$ & {$[10]$} \\
\hline Psicologo/Psicoterapeuta (cod. 89.7) & $€ 20,66$ & {$[10]$} \\
\hline Giorni lavorativi 2017 & 250 & $\begin{array}{l}\text { http://www.giorni-lavorativi.com/ } \\
\text { giorni-lavorativi_festivi_20I7.htm }\end{array}$ \\
\hline Legge $289 / 90$ & $€ 279,47$ & $\begin{array}{l}\text { https://www.inps.it/nuovoportaleinps/ } \\
\text { default.aspx?itemdir=4348I }\end{array}$ \\
\hline
\end{tabular}

L'84,8\% dei pazienti appartenenti al campione è risultato usufruire dell'assistenza da parte di un caregiver. I caregivers occupati sono risultati pari al $53 \%$ e tra questi, $1^{\prime} 86,8 \%$ ha dichiarato di aver perso giornate di lavoro a causa della prestazione di assistenza. La percentuale di caregivers non occupati a causa della prestazione di assistenza è risultata pari al 7\%.

Con riferimento ai soli pazienti e caregivers occupati sono state stimate rispettivamente il numero medio di giornate lavorative perse negli ultimi tre mesi a causa della malattia $(5,2)$ e a causa della prestazione di assistenza $(12,8)$. Relativamente ai soli pazienti occupati sono state infine stimate il numero medio di giornate lavorative con una ridotta capacità produttiva negli ultimi tre mesi $(2,2)$.

\section{Costi diretti}

\section{Costi diretti a carico del Servizio Sanitario Nazionale}

Nel questionario i pazienti sono stati chiamati a indicare il numero di volte in cui hanno usufruito, nell'ultimo anno, di servizi sanitari i cui costi sono stati sostenuti o rimborsati dal SSN. Il costo medio annuo per singolo paziente affetto da SMA sostenuto dal SSN è stato ottenuto moltiplicando il numero medio di volte in cui il paziente ha dichiarato di aver usufruito dei servizi sanitari rimborsati dal SSN per la tariffa nazionale corrispondente a ciascuna voce di costo. ${ }^{8}$
I costi medi di ciascun paziente sono stati sommati e, successivamente, redistribuiti sul totale di partecipanti allo studio restituendo così il costo medio per paziente SMA.

In media, i costi diretti sostenuti dal SSN per un paziente affetto da SMA sono risultati pari a $€ 1.878,83$. Il 97\% di questi costi è risultato attribuibile alle visite specialistiche $(€ 1.815,45)$, mentre il restante $3 \%(€ 63,38)$ è risultato associato a esami e prestazioni.

\section{Costi diretti a carico del paziente}

Nel questionario i pazienti sono stati chiamati a indicare la spesa media annua sostenuta in corrispondenza di alcune voci di costo relative ai servizi sanitari che il SSN non rimborsa, la cosiddetta spesa out-of-pocket. La spesa media annua totale sostenuta da un paziente affetto da SMA per i servizi non rimborsati dal SSN è risultata pari a $€ 2.578,14$; circa il $66 \%$ di tale spesa è risultato attribuibile al costo delle attrezzature mediche al netto del rimborso statale $(€ 1.697,61)$, circa il $18 \%$ è risultato attribuibile alla spesa sostenuta per gli esami e le visite specialistiche $(€ 466,06)$, mentre circa il $16 \%$ è risultato associato alla spesa sostenuta per le figure professionali (€414,47).

Ai pazienti è stato inoltre richiesto di quantificare la spesa media annua sostenuta per apportare eventuali modifiche all'abitazione o trasferirsi in una nuova casa accessibile e per adattare l'automobile o comprare una nuova macchina. I costi per apportare tali modifiche a carico 
Tabella 2. Caratteristiche generali della popolazione oggetto di studio.

\begin{tabular}{|c|c|c|c|c|c|c|}
\hline Totale campione & M & $\%$ & $\mathrm{~F}$ & $\%$ & Totale campione & \\
\hline $\begin{array}{l}\text { Sesso } \\
\text { (\% di non risposta } 4,2 \text { ) }\end{array}$ & 48 & $40,68 \%$ & 65 & $55,08 \%$ & I I8 (5 non rispondenti) & \\
\hline Età & M & & $\mathrm{F}$ & & Totale campione & \\
\hline $\begin{array}{l}\text { Età media }(\mathrm{ds}) \\
\text { (\% di non risposta } 4,2)\end{array}$ & $19,6(19,1)$ & & $18,4(16,6)$ & & $18,5(17,4)$ & \\
\hline $\begin{array}{l}\text { Età media alla diagnosi (ds) } \\
\text { (\% di non risposta } 3,3 \text { ) }\end{array}$ & $3,4(6,7)$ & & $2,5(6,5)$ & & $2,9(6,4)$ & \\
\hline Tipo SMA & M & & $\mathrm{F}$ & $\begin{array}{l}\text { Nessuna } \\
\text { risposta }\end{array}$ & Totale campione & \\
\hline SMA I & 12 & & 14 & I & 27 & \\
\hline SMA II & 20 & & 34 & 3 & 57 & \\
\hline SMA III & 16 & & 17 & I & 34 & \\
\hline Titolo di studio del paziente maggiorenne & M & $\%$ & $\mathrm{~F}$ & $\%$ & Totale campione & $\%$ \\
\hline Nessun titolo & 0 & $0,0 \%$ & 0 & $0,0 \%$ & 0 & $0,0 \%$ \\
\hline Licenza elementare & 0 & $0,0 \%$ & 0 & $0,0 \%$ & 0 & $0,0 \%$ \\
\hline Licenza media & 4 & $21,1 \%$ & 2 & $10,0 \%$ & 6 & $15,4 \%$ \\
\hline Diploma 2/3 anni & 0 & $0,0 \%$ & I & $5,0 \%$ & 1 & $2,6 \%$ \\
\hline Diploma 5 anni & 12 & $63,2 \%$ & 10 & $50,0 \%$ & 22 & $56,4 \%$ \\
\hline Laurea/Dottorato & 3 & $15,8 \%$ & 6 & $30,0 \%$ & 9 & $23,1 \%$ \\
\hline Nessuna risposta & 0 & $0,0 \%$ & I & $5,0 \%$ & I & $2,6 \%$ \\
\hline Totale & 19 & $100,0 \%$ & 20 & $100,0 \%$ & 39 & $100,0 \%$ \\
\hline Stato lavorativo del paziente maggiorenne & M & $\%$ & $\mathrm{~F}$ & $\%$ & Totale campione & $\%$ \\
\hline Occupato/a & 6 & $31,6 \%$ & 7 & $35,0 \%$ & 13 & $33,3 \%$ \\
\hline Disoccupato/a & 2 & $10,5 \%$ & 2 & $10,0 \%$ & 4 & $10,3 \%$ \\
\hline Studente/ssa & 3 & $15,8 \%$ & 5 & $25,0 \%$ & 8 & $20,5 \%$ \\
\hline In cerca di prima occupazione & I & $5,3 \%$ & 0 & $0,0 \%$ & 1 & $2,6 \%$ \\
\hline Inabile al lavoro & 0 & $0,0 \%$ & 3 & $15,0 \%$ & 3 & $7,7 \%$ \\
\hline Ritirato/a dal lavoro & I & $5,3 \%$ & 0 & $0,0 \%$ & 1 & $2,6 \%$ \\
\hline Non occupato/a a causa della malattia & 4 & $21,1 \%$ & 2 & $10,0 \%$ & 6 & $15,4 \%$ \\
\hline Casalinga & 0 & $0,0 \%$ & 0 & $0,0 \%$ & 0 & $0,0 \%$ \\
\hline Altro & 2 & $10,5 \%$ & 1 & $5,0 \%$ & 3 & $7,7 \%$ \\
\hline Nessuna risposta & 0 & $0,0 \%$ & 0 & $0,0 \%$ & 0 & $0,0 \%$ \\
\hline Totale & 19 & $100,0 \%$ & 20 & $100,0 \%$ & 39 & $100,0 \%$ \\
\hline
\end{tabular}

SMA: spinal muscular atrophy, atrofia muscolare spinale; ds: deviazione standard.

di un paziente affetto da SMA sono risultati mediamente pari a $€ 12.113,05$.

\section{Costi indiretti}

\section{Assenteismo, presenteismo e disoccupazione dei pazienti}

Utilizzando l'informazione circa il reddito annuo dei pazienti occupati e il numero di giornate lavorative perse da questi ultimi a causa della malattia è stato possibile ottenere una quantificazione dei costi associati all'assenteismo relativamente a ciascun paziente. Agli intervistati che hanno preferito non rispondere alla domanda sul reddito annuo percepito è stato attribuito il reddito medio annuo calcolato sulla base delle risposte fornite e inerenti ai pazienti occupati appartenenti al campione. Dividendo il reddito annuo per il numero di giornate lavorative presenti in un anno è stata ottenuta una stima del reddito medio giornaliero per ciascun paziente. Il costo per assenteismo a livello di singolo paziente è stato ottenuto moltiplicando il reddito medio giornaliero per il numero di giornate perse a causa della malattia dichiarato da ciascun intervistato. Sommando i costi indiretti per assenteismo associati ai pazienti occupati appartenenti al campione e redistribuendo tale somma per il numero di rispondenti al questionario, il costo medio annuo associato all'assenteismo per un paziente affetto da SMA è risultato pari a $€ 197,96$.

Con riferimento al presenteismo, moltiplicando il reddito medio giornaliero di ciascun paziente occupato per 
Tabella 3. Parametri per il calcolo dei costi indiretti.

\begin{tabular}{|c|c|c|c|c|}
\hline \multicolumn{4}{|l|}{ Altre variabili } & Stima \\
\hline \multicolumn{4}{|c|}{ Percentuale dei pazienti occupati che hanno perso giornate di lavoro } & $53,9 \%$ \\
\hline \multicolumn{4}{|c|}{ Percentuale dei pazienti occupati con giorni di lavoro caratterizzati da una ridotta capacità produttiva } & $23,1 \%$ \\
\hline \multicolumn{4}{|c|}{ Percentuale della ridotta capacità produttiva media sul lavoro da parte dei pazienti occupati } & $28,5 \%$ \\
\hline \multicolumn{4}{|l|}{ Percentuale dei pazienti che usufruiscono del caregiver } & $84,8 \%$ \\
\hline \multicolumn{4}{|c|}{$\begin{array}{l}\text { Percentuale dei caregivers occupati che hanno perso giornate di lavoro a causa della prestazione di } \\
\text { assistenza }\end{array}$} & $86,8 \%$ \\
\hline Giornate di lavoro perse/con ridotta capacità produttiva & Stima & Min & Max & Deviazione standard \\
\hline $\begin{array}{l}\text { Numero medio di giornate di lavoro perse negli ultimi } \\
\text { tre mesi dal paziente occupato a causa della malattia }\end{array}$ & 5,2 & 0,0 & 30,0 & 8,62 \\
\hline $\begin{array}{l}\text { Numero medio di giornate di lavoro con una ridotta } \\
\text { capacità produttiva negli ultimi tre mesi per un paziente } \\
\text { occupato a causa della malattia }\end{array}$ & 2,2 & 0,0 & 15,0 & 4,80 \\
\hline $\begin{array}{l}\text { Numero medio di giornate di lavoro perse per negli } \\
\text { ultimi tre mesi da parte del caregiver a causa della } \\
\text { prestazione di assistenza }\end{array}$ & 12,8 & 0,0 & 60,0 & 13,20 \\
\hline
\end{tabular}

il numero medio di giornate con una ridotta capacità produttiva e per la percentuale di ridotta capacità produttiva da lui dichiarati, è stato ottenuto il costo per presenteismo per ogni singolo paziente. Sommando i costi per presenteismo di ciascun paziente e redistribuendo tale somma per il numero di partecipanti allo studio, il costo medio annuo associato al presenteismo per un paziente affetto da SMA è risultato pari a $€ 59,32$.

Considerando invece i pazienti che hanno dichiarato di non essere occupati a causa della malattia $(15,38 \%$ dei rispondenti), si è assunto che questi pazienti abbiano avuto una perdita di produttività annua pari al reddito complessivo dichiarato dagli occupati $(€ 23.845,77)$. La stessa assunzione è stata effettuata per i pazienti che hanno dichiarato di aver abbandonato il lavoro a causa della malattia $(12,82 \%)$, escludendo però da quest'ultimo calcolo quelli che avessero già dichiarato di essere disoccupati a causa della malattia (al fine di evitare un doppio conteggio di perdita di produttività sullo stesso paziente). Sommando i costi per disoccupazione di ciascun paziente è stato ottenuto il costo totale per disoccupazione del campione il quale, redistribuito per il totale di partecipanti, ha fornito il costo medio annuo per paziente affetto da SMA, pari a $€ 2.020,83$.

\section{Assenteismo e disoccupazione dei caregivers}

Al fine di stimare i costi indiretti per assenteismo dei caregivers, il reddito annuo di ciascun caregiver occupato è stato diviso per i giorni lavorativi presenti in un anno al fine di ottenere il reddito medio giornaliero. Agli intervistati che hanno preferito non rispondere alla domanda sul reddito annuo percepito dal caregiver è stato attribuito il reddito medio annuo calcolato sulla base delle risposte fornite e inerenti ai caregivers occupati appartenenti al campione.
Il costo a livello di singolo caregiver è stato ottenuto moltiplicando tale reddito medio giornaliero per il numero di giornate lavorative perse dal caregiver occupato a causa della prestazione di assistenza; sommando i costi indiretti per assenteismo del caregiver relativamente ai pazienti che hanno dichiarato di usufruire dell'assistenza informale privata e redistribuendo tale somma per il numero di rispondenti al questionario, il costo medio annuo associato all'assenteismo del caregiver per un paziente affetto da SMA è risultato pari a $€ 2.172,37$.

Con riferimento alla stima del costo per disoccupazione del caregiver si è proceduto, come nel caso del paziente, considerando solo i caregivers che avessero dichiarato di essere non occupati a causa dell'assistenza o di aver abbandonato l'attività lavorativa a causa della prestazione di assistenza. Per tali caregivers si è assunta una perdita di produttività pari al reddito medio annuo dichiarato dai caregivers occupati, ossia pari a $€ 23.490,15$. Il costo medio per un paziente affetto da SMA per disoccupazione del caregiver, ottenuto sommando i costi relativi a ciascun caregiver e redistribuendo tale somma sul totale dei partecipanti, è risultato pari a $€ 5.773,00$.

\section{Costi previdenziali}

Ai partecipanti allo studio è stato chiesto di indicare se usufruissero dei benedici derivanti dalla Legge 289/90 relativa all'indennità di frequenza; tale legge consiste in un contributo economico mensile pari a $€ 279,47$ (per l'anno $2017)^{13}$ che viene corrisposto agli invalidi civili minori d'età, il cui fine è quello di fornire un sostegno economico ai fini dell'inserimento scolastico e sociale. Nell'analisi si è assunta una fruizione minima del contributo (pari a nove mesi) per i pazienti minorenni (67\% del campione) il cui caregiver ne avesse dichiarato la fruizione $(23 \%$ degli 
Tabella 4. Costi medi per paziente per tipologia di SMA e tipologia di costo.

\begin{tabular}{|c|c|c|c|c|}
\hline Tipologia di costo & $\begin{array}{l}\text { Costo medio/pz } \\
\text { SMA I } \\
N=27\end{array}$ & $\begin{array}{l}\text { Costo medio/pz } \\
\text { SMA II } \\
N=57\end{array}$ & $\begin{array}{l}\text { Costo medio/pz } \\
\text { SMA III } \\
N=34\end{array}$ & $\mathrm{p}$-value \\
\hline Costi diretti (SSN) & $€ 3.385,49$ & $€ I .828,23$ & $€ 767,20$ & 0,0168 \\
\hline Visite specialistiche & $€ 3.3 \mid 4,02$ & $€ I .768,42$ & $€ 704,26$ & 0,0146 \\
\hline Esami/prestazioni & $€ 7 I, 47$ & $€ 59,81$ & $€ 62,94$ & 0,9525 \\
\hline Costi diretti (a carico del paziente) & $€ I .244,8 \mid$ & $€ 3.414,82$ & $€ 2.234,29$ & 0,0609 \\
\hline Costo per esami e visite specialistiche & $€ 436,48$ & $€ 463,70$ & $€ 493,50$ & 0,4248 \\
\hline Costo per attrezzature mediche & $€ 366,96$ & $€ 2.396,54$ & $€ I .582,56$ & 0,0182 \\
\hline Figure professionali & $€ 44 I, 37$ & $€ 554,58$ & $€ I 58,24$ & 0,7561 \\
\hline Costi indiretti & $€ \mid 3.053,55$ & $€ 10.73 \mid, 73$ & $€ 9.522,03$ & 0,5359 \\
\hline Assenteismo paziente & $€ 0,00$ & $€ 280,70$ & $€ 216,47$ & 0,3049 \\
\hline Presenteismo paziente & $€ 0,00$ & $€ 105,96$ & $€ 28,23$ & 0,6273 \\
\hline Disoccupazione paziente & $€ 0,00$ & $€ 1.673,39$ & $€ 4.208,08$ & 0,0431 \\
\hline Assenteismo caregiver & $€ 1.543,66$ & $€ 2.431,26$ & $€ 2.237,62$ & 0,5168 \\
\hline Disoccupazione caregiver & $€|| .310,07$ & $€ 5.769,5 \mathrm{I}$ & $€ \mathrm{I} .38 \mathrm{I}, 77$ & 0,0008 \\
\hline Costi previdenziali L.289/90 e L. $104 / 92$ & $€ 199,82$ & $€ 470,91$ & $€ I .449,86$ & 0,0054 \\
\hline Costi per modifiche all'auto e all'abitazione & $€ 17.998,97$ & $€ \mid 4.435,76$ & $€ 3.545,00$ & 0,0413 \\
\hline
\end{tabular}

pz: paziente; SSN: Servizio Sanitario Nazionale; SMA: spinal muscular atrophy, atrofia muscolare spinale.

aventi diritto). Il costo previdenziale medio annuo per un paziente affetto da SMA è risultato pari a $€ 383,68$.

È stato inoltre chiesto ai partecipanti, se usufruissero dei benefici derivanti dalla Legge 104/92, la quale prevede che il caregiver del paziente la cui invalidità sia stata accertata usufruisca di quattro giorni al mese di permesso retribuito per l'assistenza fornita al proprio familiare. A livello di singolo paziente, ai fini del calcolo dei costi previdenziali relativi a tale legge, sono stati considerati i pazienti che avessero dichiarato di usufruirne ( $82 \%$ del totale dei rispondenti al questionario) e, tra questi, quelli che avessero dichiarato di usufruire dell'assistenza del caregiver occupato che non avesse già dichiarato di aver avuto giorni di assenteismo (al fine di evitare un doppio conteggio dovuto alla possibilità che il caregiver avesse già contato i giorni relativi alla Legge 104/92 nei giorni di assenteismo). La percentuale di rispondenti che soddisfa tutte queste condizioni è risultata pari al $6 \%$ dei rispondenti che hanno dichiarato di usufruire dei benefici relativi alla legge in questione. A ciascuno di tali rispondenti è stata attribuita una perdita di produttività associata alla Legge 104/92 pari al reddito giornaliero del rispondente moltiplicato per 4 e successivamente per 12 al fine di ottenere il costo annuale. Sommando i costi associati a ciascuno di questi rispondenti e redistribuendo il totale per il numero di partecipanti allo studio, si è ottenuto il costo medio annuo per paziente affetto da SMA associato alla Legge 104/92, il quale è risultato pari a $307,27 €$.

\section{Costi medi per paziente e per tipologia di SMA}

In Tabella 4 sono riportati i costi medi per paziente per tipologia di SMA e per voce di costo.
Con riferimento ai costi diretti a carico del SSN, il costo medio più elevato è risultato associato ai pazienti con SMA di tipo I (€3.385,49); segue il costo associato ai pazienti con SMA di tipo II $(€ 1.828,23)$ e il costo associato ai pazienti con SMA di tipo III $(€ 767,20)(p=0,0168)$. Il costo medio più elevato in termini di costi diretti a carico del paziente è risultato associato ai pazienti con SMA di tipo $2(€ 3.414,82)$; per i pazienti con SMA di tipo II e III, il $70 \%$ dei costi diretti a carico del paziente è risultato associato al costo sostenuto per le attrezzature mediche (rispettivamente, pari a $€ 2.396,54$ e $€ 1.582,56$ ), mentre ai pazienti con SMA di tipo I sono risultati associati i costi più bassi $(€ 366,96)(\mathrm{p}=0,0182)$.

Relativamente ai costi indiretti, ai pazienti con SMA di tipo I è risultato associato il costo medio per disoccupazione del caregiver più elevato $(€ 11.310,07)$; seguono i costi associati al paziente con SMA di tipo II $(€ 5.769,51)$ e i costi associati al paziente con SMA di tipo III $(€ 1.381,77)$ $(p=0,0008)$.

I costi indiretti medi per paziente risultano la voce di costo più elevata per ciascuna tipologia di SMA (Figura 2). In Tabella 5 sono riportati i costi medi per paziente con SMA in relazione a ciascuna caratteristica demografica rilevata tramite il questionario.

\section{Discussione}

L'obiettivo degli studi di costo della malattia è quello di fornire una misura economica dell'impatto della malattia oggetto di studio, ma affinché si abbia una corretta valutazione dell'impatto di una patologia è importante includere tutte le possibili voci di costo a essa riconducibili. Dunque, andare oltre la sola quantificazione dei costi sostenuti dal SSN e fornire anche una misura dei costi 


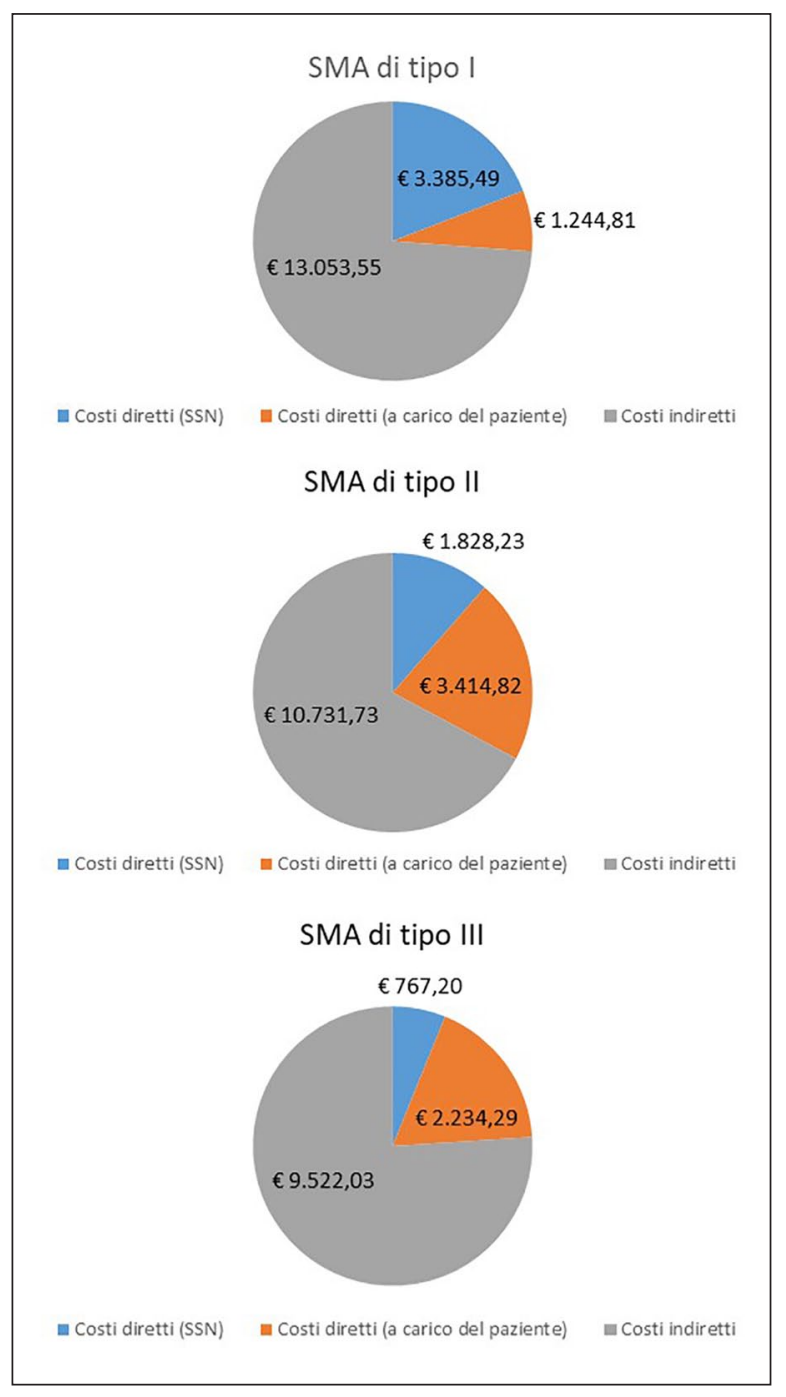

Figura 2. Costi medi per paziente per voce di costo e tipologia di SMA.

indiretti e dei costi sostenuti dalle famiglie rappresenta un aspetto cruciale nella comprensione del peso economico della SMA.

Il nostro lavoro ha stimato un costo medio per paziente SMA pari a $€ 15.371$ annui mostrando come il costo indiretto della perdita di produttività sia la principale voce di spesa che grava sulla società $(67 \%$ della spesa complessiva considerata). Inoltre, il peso della malattia non grava solo sui pazienti ma anche, e a volte soprattutto, sui caregiver e sulle famiglie $(76 \%$ dei costi per pazienti SMA I sono costi indiretti dei caregiver).

Assumendo che il campione analizzato sia rappresentativo della popolazione SMA italiana, e considerando un numero di persone attualmente affette dalla patologia in Europa compreso tra $1,31-1,87$ su 100 mila individui ${ }^{7}$ possiamo stimare un costo medio annuo nella prospettiva sociale italiana pari a $€ 13,2$ milioni per la gestione e la cura di circa 850 pazienti. Di questi, il 67\% dei costi caratterizzati da costi indiretti ( $€ 8,7$ milioni). All'interno dei costi indiretti, oltre $€ 6,8$ milioni sono associati alla predita di produttività dei caregiver $\mathrm{e}$, in particolare, alla disoccupazione $\mathrm{e}$ all'impossibilità di partecipare all'attività produttiva del paese a causa della malattia dell'assistito $(73 \%$ dei costi indiretti).

Se consideriamo il costo sostenuto dalle famiglie, ogni anno in Italia le famiglie SMA spendono oltre $€ 2,2$ milioni di tasca propria per la gestione della malattia. Mediamente, oltre $€ 2$ mila annui, quasi due stipendi mensili, vengono assorbiti dalla gestione quotidiana della malattia. Di questi, il $66 \%$ è costituito dai costi sostenuti per l'utilizzo di attrezzature mediche (riportate al netto del rimborso statale), il 18\% dai costi per esami e visite specialistiche il cui costo non è sostenuto o rimborsato dal SSN e il $16 \%$ dai costi sostenuti per figure professionali di cui il paziente si avvale al di fuori delle strutture ospedaliere.

Considerando la prospettiva del solo SSN, il modello economico stima una spesa annua pari a circa $€ 1,6$ milioni, il $97 \%$ dei quali è risultato associato ai costi per le visite specialistiche. Ovviamente, questi valori sono molto sottostimati dato che nel computo complessivo non sono stati considerati i costi delle ospedalizzazioni generate dalla malattia e dei nuovi farmaci per il trattamento della patologia.

Una particolare voce di spesa riguardano i costi per modifiche all'auto e all'abitazione che, all'interno dell'analisi, sono state considerate separatamente essendo una spesa ammortizzabile nel corso degli anni e non necessariamente stimabile in termini annui. Il modello, ipotizzando un ammortamento della spesa sulla base del tempo trascorso dalla diagnosi al momento dell'intervista, ha stimato un peso economico per la gestione straordinaria di casa e auto pari a circa $€ 10,2$ milioni.

A nostra conoscenza, questo studio rappresenta il primo tentativo di quantificare i costi sostenuti in Italia dai pazienti affetti da SMA e dai loro caregivers. A livello europeo, uno studio condotto in Germania nel $2013^{4}$ ha evidenziato un costo medio annuo per paziente pari a $€ 70.566$, quasi cinque volte superiore rispetto a quello stimato dal presente studio. Tuttavia, nel complesso, la ripartizione delle voci di costo incluse nella presente analisi risulta coerente (58\% dei costi totali era a carico del paziente, il $22 \%$ risultava derivante dalla perdita di produttività e il $20 \%$ risultava associato ai costi sanitari non a carico del paziente) mostrando come i costi indiretti siano altamente impattanti per famiglie che si trovano ad affrontare la malattia.

Il presente studio non è esente da limiti e va interpretato considerandone le principali criticità. Il primo limite riguarda la numerosità campionaria in quanto questa risulta limitata, ma trattandosi di una patologia rara risulta difficile reperire un campione numeroso. In secondo luogo, la mancata inclusione di alcune voci di costo (costi relativi al trasporto per recarsi alle visite o nelle strutture 
Tabella 5. Costi medi per paziente con SMA stratificati per le caratteristiche demografiche del campione.

\begin{tabular}{|c|c|c|c|}
\hline & $\mathbf{N}$ & Costo medio/paziente & p-value \\
\hline Totale campione & 118 & $€|5.37|, 4 \mid$ & \\
\hline \multicolumn{4}{|l|}{ Sesso } \\
\hline Maschio & 48 & $€ \mid 6.096,55$ & \\
\hline Femmina & 65 & $€ \mid 4.804,70$ & 0,841 \\
\hline Nessuna risposta & 5 & $€ \mid 5.777,37$ & \\
\hline \multicolumn{4}{|l|}{ Età } \\
\hline Età [0-18] & 75 & $€ \mid 2.835,49$ & \\
\hline Età [18-39] & 18 & $€|5.66|, 06$ & 0,349 \\
\hline Età [39-80] & 25 & $€ 22.770,62$ & 0,002 \\
\hline \multicolumn{4}{|l|}{ Titolo di studio } \\
\hline Diploma scuola secondaria superiore & 22 & $€|8.3| 8,84$ & \\
\hline Diploma 2-3 anni & 1 & $€ 35.855,28$ & 0,472 \\
\hline Laurea breve, laurea, dottorato & 9 & $€ \mid 2.625,63$ & 0,303 \\
\hline Licenza media & 6 & $€ 21.535,0 \mathrm{I}$ & 0,700 \\
\hline Nessuna risposta & 80 & $€|4.15|, 45$ & \\
\hline \multicolumn{4}{|l|}{ Stato lavorativo del paziente } \\
\hline Occupato/a & 13 & $€ \mid I .787,68$ & \\
\hline Altro & 3 & $€ 24.964,86$ & 0,097 \\
\hline Disoccupato/a & 4 & $€ 3.075,00$ & 0,004 \\
\hline In cerca di prima occupazione & 1 & $€ 0,00$ & - \\
\hline Inabile al lavoro & 3 & $€ 40.769,25$ & 0,008 \\
\hline Non occupato/a a causa della malattia & 6 & $€ 38.236,84$ & 0,001 \\
\hline Ritirato/a dal lavoro & I & $€ 5.166,35$ & 0,354 \\
\hline Studente/ssa & 8 & $€ I 5.204,92$ & 0,343 \\
\hline Nessuna risposta & 79 & $€ 13.858,96$ & \\
\hline
\end{tabular}

adibite alla terapia richiesta, costi di consulenza legale, costi associati a eventuali diete speciali, o a prestazioni sanitarie non presenti nel questionario somministrato, ecc.), ha generato dei risultati che molto probabilmente tenderanno a sottostimare il peso economico complessivo.

\section{Conclusioni}

Lo studio ha evidenziato un costo medio annuo per un paziente affetto da SMA in Italia pari a $€ 15.371,41$ (a esclusione dei costi medi per modifiche all'auto e all'abitazione per paziente pari a $€ 12.113,05)$.

Il costo medio stimato è costituito per il $67 \%$ $(€ 10.233,48)$ da costi indiretti calcolati in termini di perdita di produttività sul lavoro, per il 4\% $(€ 690,95)$ da costi previdenziali, i costi a carico del paziente (esclusi i costi per modifiche auto e abitazione) costituiscono il $17 \%$ $(€ 2.578,14)$ del costo medio totale, mentre quelli a carico del SSN costituiscono il 12\% (€1.878,83).

In conclusione, la SMA produce in Italia notevoli costi non facilmente visibili in quanto costituiti per la maggior parte da costi a carico del paziente e da costi indiretti valutati in termini di perdita di produttività sul lavoro. L'obiettivo di questo lavoro è stato proprio quello di fornire una misura di tali costi che andasse a colmare una lacuna dovuta alla scarsità di studi effettuati sulla patologia a livello internazionale, e una completa assenza degli stessi a livello nazionale. Questo studio potrebbe dunque costituire un valido supporto per i decisori e per la valutazione economica di nuovi ipotetici trattamenti, i quali molto spesso non vengono valutati nel caso di malattie rare, a causa della bassa incidenza di queste ultime.

\section{Declaration of Conflicting Interest}

The authors declare that there is no conflict of interest.

\section{Funding}

The author(s) disclosed receipt of the following financial support for the research, authorship, and/or publication of this article: This study is supported by Biogen Srl Italia.

\section{ORCID iDs}

Andrea Marcellusi (iD) https://orcid.org/0000-0002-3974-3798 Maria A. Rotundo (iD https://orcid.org/0000-0002-4016-0055

\section{Bibliografia}

1. Associazione Famiglie SMA. http://www.famigliesma.org/ quante-comune-la-sma/ (ultimo accesso 28 luglio 2019).

2. Munsat TL and Davies K. International SMA Consortium meeting, 26-28 June 1992, Bonn, Germany. Neuromusc Disord. 1992; 2: 423-428. 
3. Faravelli I, Nizzardo M, Comi GP, et al. Nat Rev Neurol. 2015; 11: 351-359.

4. Klug C, Schreiber-Katz O, Theile S, et al. Disease burden of spinal muscular atrophy in Germany. Orphanet J Rare Dis. 2016; 11(1): 58 .

5. Fabriani V, etal. Cost of Illness Analysis ofDuchenne Muscular Dystrophy In Italy. Value in Health. 2013; 17(7): A528.

6. Mennini FS, et al. Pilot evaluation of indirect costs and the impact of bipolar disorder type I. Journal of Psychopathology. 2014; 20: 216-222.

7. Jones C, Oskoui M, Zielinski D, et al. Systematic review of incidence and prevalence of spinal muscular atrophy (SMA). Eur J Paediatr Neurol. 2015; 19 (Suppl 1): S65-S65.

8. Decreto del Ministero della Salute dell'18 ottobre 2012. Nomenclatore tariffario dell'assistenza specialistica ambulatoriale. http://www.salute.gov.it/portale/temi/p2_6. jsp?lingua $=$ italiano $\& i d=1767 \&$ area $=$ programmazioneSanit ariaLea\&menu=lea (ultimo accesso 28 luglio 2019).

9. Giorni lavorativi presenti in un anno. http://www.giornilavorativi.com/giorni-lavorativi_festivi_2017.htm (ultimo accesso 29 luglio 2019).
10. Ministero della Salute. Remunerazione prestazioni di assistenza ospedaliera per acuti, assistenza ospedaliera di raibilitazione e di lungodegenza post acuzie e di assistenza specialistica ambulatoriale. GU Serie Generale n. 23 del 28/01/2013 Suppl. Ordinario n. 8. https://www .gazzettaufficiale.it/eli/id/2013/01/28/13A00528/sg (ultimo accesso 28 luglio 2019).

11. Barber J and Thompson S. Multiple regression of cost data: use of generalised linear models. J Health Serv Res Policy. 2004; 9(4): 197-204.

12. Mennini FS, Viti R, Bini $\mathrm{C}$, et al. Economic burden of disease of uncontrolled acromegalic patients: the acromegaly Italian collaborative study group. Global \& Regional Health Technology Assessment. 2018. https://doi .org/10.1177/2284240318755065.

13. INPS (Istituto Nazionale di Previdenza Sociale) Invalidi civili - prestazioni economiche / Indennità mensile di frequenza. https://www.inps.it/nuovoportaleinps/default. aspx?sPathID=\%3b0\%3b45138\%3b45545\%3b45566\%3b4 $5585 \% 3 \mathrm{~b} 45589 \% 3 \mathrm{~b} \&$ lastMenu $=45589 \& \mathrm{iMenu}=1 \& \mathrm{iNodo}=$ $45589 \& \mathrm{p} 4=2$. 


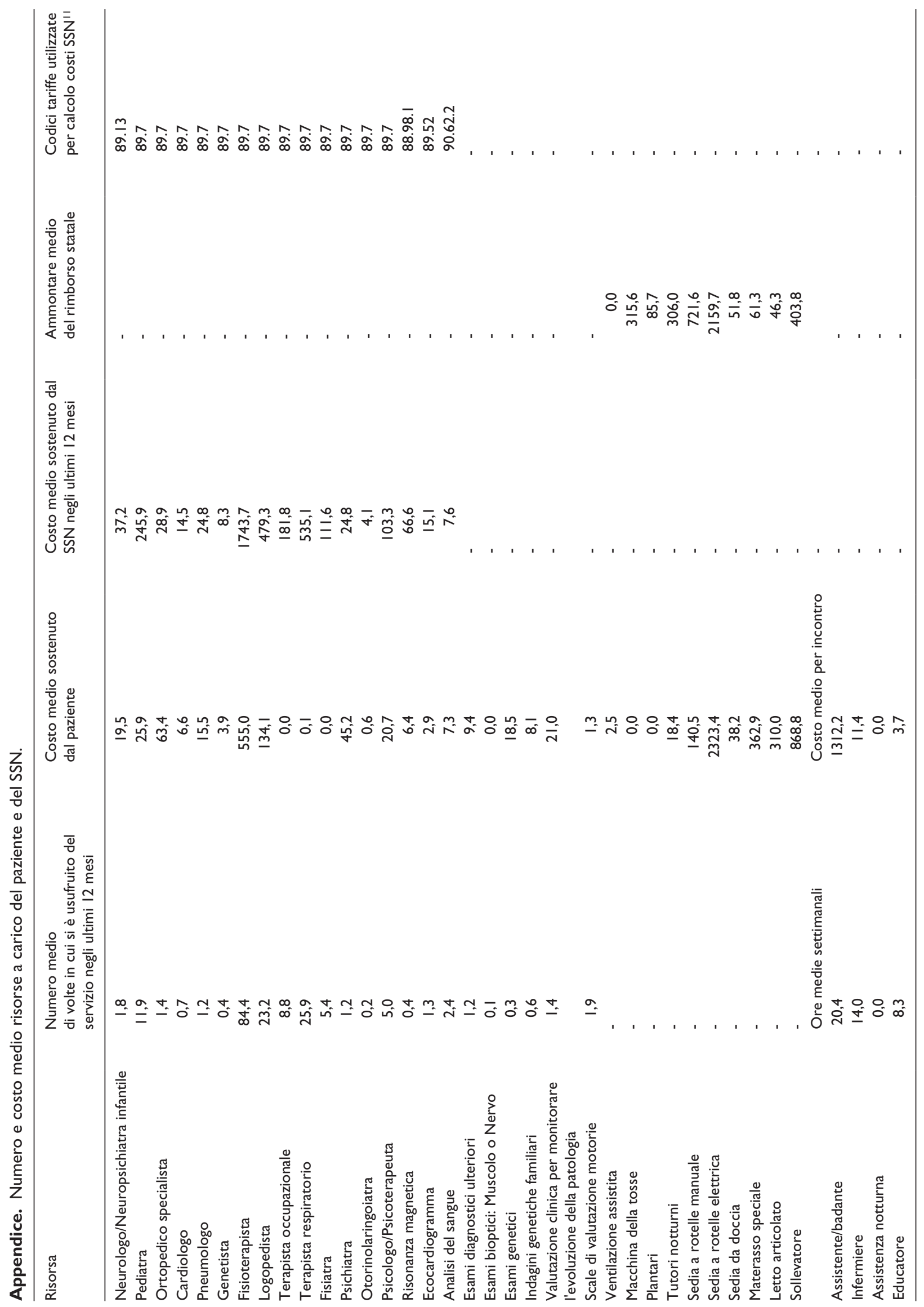

